\section{PCJM2: A program for the axiomatic conjoint measurement analysis of polynomial composition rules}

\section{JAMES R. ULLRICH, DAVID E. CUMMINS, and JOHN WALKENBACH \\ University of Montana, Missoula, Montana 59812}

Description. This program is designed to discriminate between the additive $[\mathrm{A}+\mathrm{P}+\mathrm{U}]$, the distributive $[(\mathrm{A}+\mathrm{P}) \mathrm{U}]$, and the dual-distributive $[\mathrm{AP}+\mathrm{U}]$ polynomial composition rules using the axiomatic tests of the algorithm described by Krantz and Tversky (1971). This revised version of the original PCJM program (Ullich \& Cummins, 1973) incorporates several substantial additions to the original routine. New additions include an option to assist in assessing "signed" cases, the ability to work with multiple entries per cell, provisions for handling missing data, and the inclusion of a count of the number of times a particular cell is involved in a violation of each of the axiomatic tests. The input consists of the number of levels of each of the three variables and a matrix of the data obtained from the factorial combinations of the three variables. The program is broken down into a number of subroutines, which analyze the data for the diagnostic tests of independence of each variable from the other two and of double cancellation and joint independence for all pairs of variables. Two additional subroutines analyze the data for distributive cancellation and dualdistributive cancellation with respect to the odd variable, which is specified upon input.

The output consists of (1) the matrix of the input data with the variables $A, P$, and $U$ identified and printed; (2) the total number of configurations of the data for which the conditions for the tests of independence, double cancellation, and joint independence are satisfied in each dimension or pair of dimensions; (3) the total number of tests for the odd variable on distributive cancellation and dual-distributive cancellation; and (4) the specific configurations representing failures of any particular test up to a user-specified maximum number, as well as the total number of failures. In addition, (5) a matrix listing the number of times each cell was involved in a violation is printed for each test. This is particularly useful in the identification of one or more "bad" data points. The number of levels of each of the three variables is limited only by the time necessary to analyze the data and the storage capacity of the particular machine. As currently dimensioned, this limit is a 6 by 6 by 6 matrix.

Program development was supported in part by a grant from the University of Montana and the University of Montana Foundation.
In addition to the intact input matrix, PCJM2 optionally performs any or all of the diagnostic tests on up to five contiguous submatrices of the original matrix. This option is especially useful for cases in which levels of one or more of the variables are zero or negative (i.e., the signed case).

PCJM2 is capable of performing the diagnostic tests when multiple cell entries are available. Specifically, from 3 to a maximum of 50 entries per cell may be input. A Mann-Whitney $U$ test is performed for all pairs of cells, and a cell is considered greater than another if the cell entries differ at less than the .05 level. Rank orders are induced and all subsequent analyses are performed on the ranks. The algorithm for this routine is the one used by Holt and Wallsten (1975).

Characteristics of the Program. The number of possible tests for which the preconditions hold is not completely specified by the algorithm as detailed by Krantz and Tversky (1971). For example, consider the test of independence specified in their Equation 1: $(a, p, u) \geqslant(b, p, u)$ if $(a, q, v) \geqslant(b, q, v)$. Literally taken, this test holds for all $a, b$ contained in $A, p, q$ contained in $P$, and $u, v$ contained in $U$. This would, however, produce a large number of trivial tests, as for example, when $a=b$. PCJM2 does not count the trivial tests, but instead will count only the desired [C(DIM 1,2)*C(DIM2*DIM3,2)] number of tests when $\mathrm{I} 1=1$, where $\mathrm{C}(\mathrm{i}, \mathrm{j})$ is the combinatorial of $\mathrm{i}$ things taken $\mathrm{j}$ at a time. A very similar restriction restricts the number of tests when checking for joint independence.

The subroutine for checking distributive cancellation has two forms of restrictions that allow it to check only the nontrivial tests of double cancellation. The first restriction is that the intermediate levels of factors $A$ and $P$ (denoted $b$ and $q$ ) must fall between the extreme values (denoted a, c, p, and r). That is, either $\mathrm{a}<\mathrm{b}<\mathrm{c}$ or $\mathrm{a}>\mathrm{b}>\mathrm{c}$ and either $\mathrm{p}<\mathrm{q}<\mathrm{r}$ or $\mathrm{p}>\mathrm{q}>\mathrm{r}$, where orderings on the lowercase alphabetic characters reflect the orderings as defined by the subroutine for checking single-factor independence.

The restrictions in the subroutine for checking distributive cancellation amount to choosing all values of the levels subject to the restriction that $u \neq v, a \neq b$, $\mathrm{c} \neq \mathrm{d}, \mathrm{p} \neq \mathrm{q}$, and $\mathrm{r} \neq \mathrm{s}$. There is an additional restriction that if $a<b$ then $c<d$ or if $a>b$ then $c>d$. Similarly, if $p<q$ then $r<s$ or if $p>q$ then $r>s$. If there is one failure in a rectangular solid picked up by the subroutine, and if there are no ties within the solid, then there will be four failures. If ties exist, there may be less than four failures. Thus, if there are no ties within the matrix, the nature of the distributive cancellation is that the number of failures must be a multiple of four. Because the presence of ties may reduce this value to less than four, it was felt not desirable to reduce this number by integer division. 
The restrictions in the subroutine to assess dualdistributive cancellation are made by a completely subjective process. They consist solely of insuring that either the order $\mathrm{a}<\mathrm{b}<\mathrm{c}<\mathrm{d}<\mathrm{e}$ or the order $a>b>c>d>e$ holds. Similarly, it must be the case that either $\mathrm{p}<\mathrm{q}<\mathrm{r}<\mathrm{s}<\mathrm{t}$ or $\mathrm{p}>\mathrm{q}>\mathrm{r}>\mathrm{s}>\mathrm{t}$ and either $u<v<w<x<y$ or $u>v>w>x>y$. The authors make no claims about the optimality of the restrictions in this subroutine. The program handles missing data in the following manner. When testing is being done, a certain number of cells are chosen by the program. If any one of these cells is missing, the test is not performed for the set of chosen cells. Missing data appear on the output listing as

Computer and Language. This program is written for the Digital Equipment Corporation System-10 computer in FORTRAN IV. No machine-specific instructions are used.
Availability. Copies of the source code, program write-up, and sample output may be obtained by sending a blank tape to James R. Ullich, Computer Center, University of Montana, Missoula, Montana 59812.

\section{REFERENCES}

Holt, J. O., \& WAllsten, T. S. A users manual for CONJOINT: A computer program for evaluating certain conjoint measurement axioms. Catalogue of Selected Documents in Psychology, 1975, 5, 317 (MS No. 1079).

Krantz, D. H., \& TyersKy, A. A conjoint-measurement analysis of composition rules in psychology. Psychological Review, 1971, 78, 151-169.

Ullrich, J. R., \& Cummins, D. E. PCJM: A program for conjoint measurement analysis of composition rules. Behavioral Science, 1973, 18, 151-169.

(Accepted for publication December 22, 1977.) 